\title{
Decreased Vision and Junctional Scotoma from Pituicytoma
}

\author{
Nancy Huynh $^{a}$ Anat O. Stemmer-Rachamimov ${ }^{b}$ \\ Brooke Swearingen $^{c}$ Dean M. Cestari ${ }^{\mathrm{a}}$ \\ ${ }^{a}$ Department of Neuro-Ophthalmology, Massachusetts Eye and Ear Infirmary, \\ Harvard Medical School, and Departments of ${ }^{b}$ Pathology and ${ }^{\mathrm{C}}$ Neurosurgery, \\ Massachusetts General Hospital, Boston, Mass., USA
}

\section{Key Words}

Junctional scotoma $\cdot$ Pituicytoma $\cdot$ Visual field defect $\cdot$ Sellar tumor $\cdot$ Optic chiasm

\begin{abstract}
Pituicytomas are rare neoplasms of the sellar region. We report a case of vision loss and a junctional scotoma in a 43-year-old woman caused by compression of the optic chiasm by a pituitary tumor. The morphological and immunohistochemical characteristics of the tumor were consistent with the diagnosis of pituicytoma. The tumor was debulked surgically, and the patient's vision improved.
\end{abstract}

\section{Introduction}

Pituicytoma is an extremely rare low-grade glioma that presumably arises from pituicytes, modified specialized glial cells located in the perivascular zones of the neurohypophysis [1]. The rarity of this tumor has contributed to its poor characterization and difficulty in diagnosis. To date, only 51 cases of pituicytomas have been reported, mostly in pathology, neurosurgery, and radiology journals. To the best of our knowledge, this is the first case of pituicytoma to be reported in the ophthalmic literature. We report a case of this rare tumor causing decreased vision and a junctional scotoma.

\section{Case Report}

A 43-year-old woman presented with progressive vision loss for 2 months. Her medical and ophthalmological histories were unremarkable. When she presented to us, her visual acuity was $20 / 15$ in the right eye, and she was able to count fingers at 2 feet in the left eye. An afferent pupillary 
defect was present in the left eye. No strabismus, motility disturbances, or proptosis was observed. Automated perimetry showed a superotemporal defect in the right eye and central scotoma in the left eye, consistent with a junctional scotoma (fig. 1a, b). Disc pallor was noted bilaterally. There was no disc edema.

Magnetic resonance imaging (MRI) of the brain with gadolinium revealed a solid, homogeneously enhancing sellar and suprasellar mass measuring $1.9 \times 1.7 \times 1.5 \mathrm{~cm}$ with compression of the optic chiasm (fig. 2a, b). Endocrinological analysis was unremarkable. Transsphenoidal surgery was attempted, but could not be achieved due to the highly vascular nature of the tumor. The tumor was debulked via a right frontotemporal craniotomy. Postoperative neuro-ophthalmologic examination showed that the visual acuity improved to $16 / 13$ in the left eye. There was no afferent papillary defect or dyschromatopsia. Repeat visual fields showed a bitemporal hemianopia (fig. 1c). Postoperative MRI with gadolinium showed that the lesion had largely been resected, measuring $1.1 \times 1.5 \times 1.0 \mathrm{~cm}$, with improvement in the displacement of the optic chiasm (fig. 2c, d). Postoperatively, the patient developed central hypothyroidism and hyperprolactinemia, and was placed on levothyroxine and cabergoline. Given the subtotal resection of the tumor, treatment with radiation was discussed but we decided on observation. At her most recent follow-up 10 months after surgery, her ophthalmologic examination, MRI results, and endocrinological status were stable.

Histopathological evaluation revealed a moderately cellular tumor composed of elongated cells with abundant eosinophilic cytoplasm arranged in a storiform pattern ( $\underline{\text { fig. } 3 \mathrm{a}}$ ). There were no mitotic figures, necrosis, Rosenthal fibers, or perivascular pseudorosette formation. Immunohistochemical stains showed that the tumor was diffusely positive for glial fibrillary acidic protein (GFAP) and focally positive for S-100 (fig. 3b, c), but negative for synaptophysin and chromogranin. These histological and immunohistochemical features raised the differential diagnosis of on cocytoma, granular cell tumor, and pituicytoma. Electron microscopy of tumor cells showed no evidence of cytoplasmic accumulations of lysosomes or mitochondria, thus supporting the diagnosis of pituicytoma (fig. 3d).

\section{Discussion}

The clinical presentation of pituicytomas is similar to that of other pituitary tumors, with patients typically presenting with visual or endocrinopathic symptoms. On neuroimaging, pituicytomas are characterized as a discrete, solid sellar mass that is isointense to the surrounding brain on $\mathrm{T}_{1}$-weighted MRI and homogeneously enhancing after gadolinium administration [2,3]. Preoperative diagnosis of pituicytomas is often challenging because of the lack of distinctive clinical or radiological features differentiating them from other pituitary tumors. Diagnosis of pituicytoma is often made only after histopathological analysis. For instance, our patient was initially suspected of having a pituitary adenoma. Only after histological and immunohistochemical analysis was it determined to be a pituicytoma.

Pathologic analysis of pituicytomas shows elongated spindle cells arranged in interlacing fascicles with little or no nuclear atypia or mitotic activity [4]. Immunohistochemistry demonstrates $\mathrm{S}-100$ and GFAP positivity, but negative staining for synaptophysin, chromogranin A and B, and neurofilament [5]. The histological and immunochemical profiles distinguish pituicytomas from other lesions of the sellar and suprasellar region, including pituitary adenoma and meningiomas. Pituitary adenomas are synaptophysin reactive, frequently chromogranin positive, and negative for GFAP and S-100. In contrast to meningiomas, pituicytomas lack whorls, psammoma bodies, and nuclear pseudo-inclusions. Differential diagnosis from other GFAP-positive sellar lesions includes pilocytic astrocytomas and diffuse astrocytomas. Pilocytic astrocytomas are composed of smaller, more elongated cells with coarse fibrillary 
processes and often have a myxoid background with scattered eosinophilic granular bodies and Rosenthal fibers. Other rare sellar lesions with a similar histological appearance are granular cell tumor and oncocytoma characterized on electron microscopy by intracytoplasmic accumulations of lysosomes (granular cell tumor) or mitochondria (oncocytoma).

Surgical resection is the mainstay of treatment for pituityctomas. In the largest series to date, Brat et al. [4] reported that, among the 6 patient who underwent total surgical resection, no recurrences of the tumor occurred. Recurrence after subtotal resection is common, with 4 of the 7 cases reported in the literature, among patients with a follow-up period of more than 6 months [4, 6-9]. Fractionated radiation therapy has been recommended for subtotal resection [9], but there is little data to suggest any benefit.

This report adds to the rare cases of pituicytoma currently in the literature and is the first case to be reported in the ophthalmic literature. Our case is also the first to report a junctional scotoma caused by pituicytoma. Ophthalmologists should become familiar with the clinical, radiological, and histopathological features of this rare neoplasm as pituicytomas can cause significant visual symptoms. 


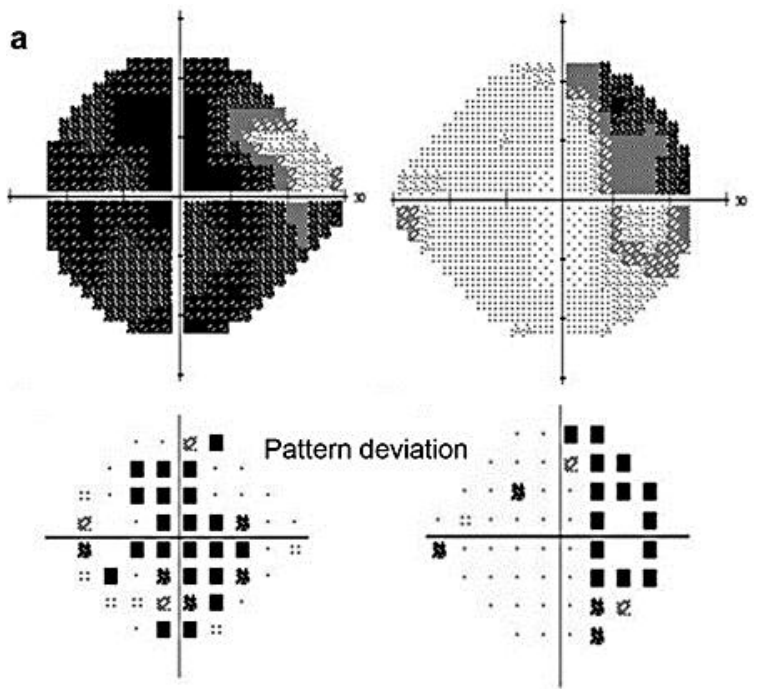

b
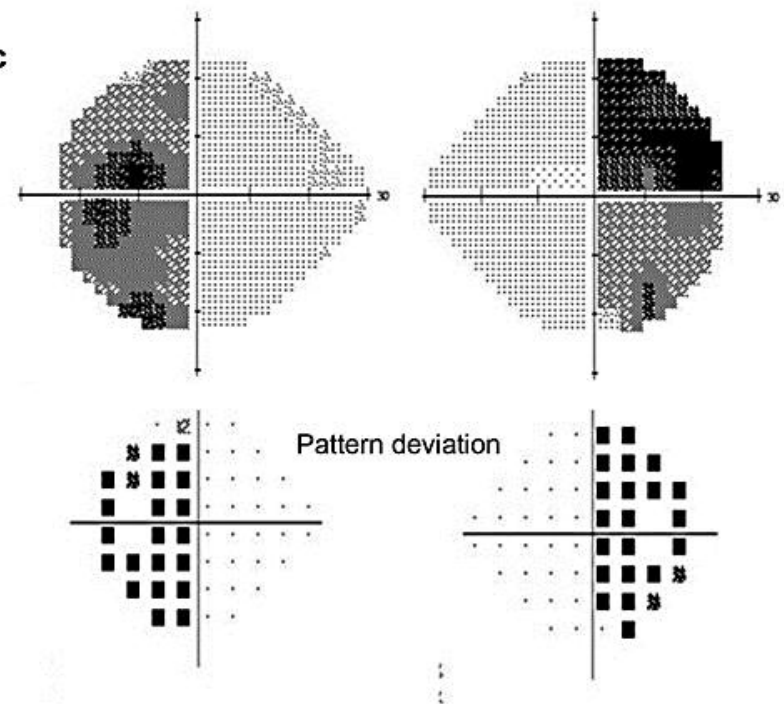

Fig. 1. a Automated visual field demonstrating a junctional scotoma. b Goldmann visual field testing of the left eye showing a central scotoma. c Postoperative visual field showing residual bitemporal hemianopia. 

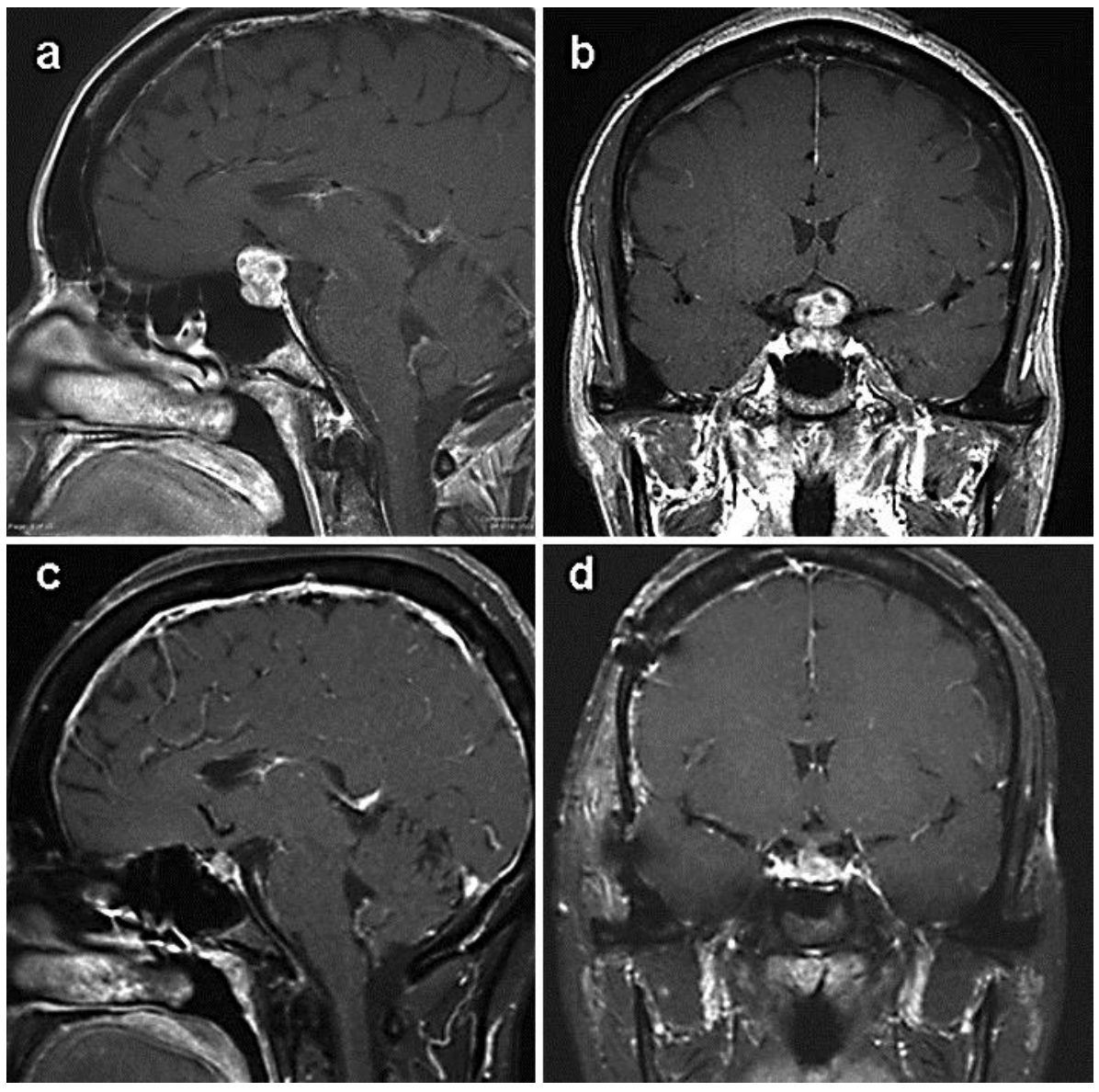

Fig. 2. Preoperative $\mathrm{T}_{1}$ sagittal (a) and coronal (b) MRI showing compression of the tumor on the optic chiasm. Postoperative $\mathrm{T}_{1}$ sagittal (c) and coronal (d) MRI showing debulking of the lesion and improvement in compression of the optic chiasm. 


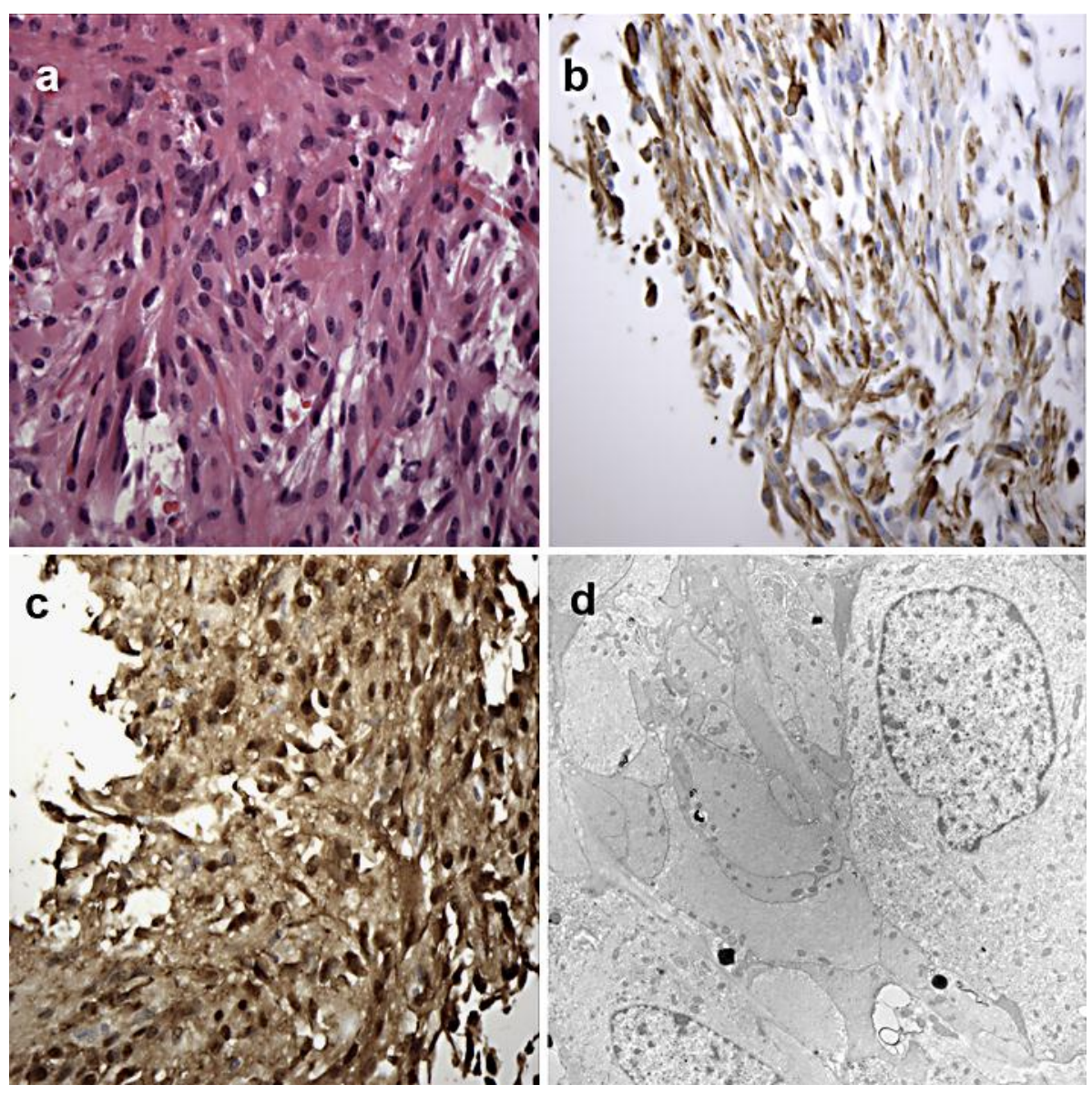

Fig. 3. Pathology of pituicytoma. a Tumor composed of spindle-shaped cells arranged in interlacing fascicle cells. HE stain, $\times 40$. b Positive glial fibrillary acidic protein staining. $\mathbf{c}$ Positive immunostaining with S-100 protein. $\mathbf{d}$ Electron microscopy shows no evidence of cytoplasmic accumulations of lysosomes or mitochondria.

\section{References}

1 Brandao RA, Braga MH, de Souza AA, Reis BL, Faraj de Lima, FB: Pituicytoma. Surg Neurol Int 2010;1:79.

2 Hammoud DA, Munter FM, Brat DJ, Pomper MG: Magnetic resonance imaging features of pituicytomas: analysis of 10 cases. J Comput Assit Tomogr 2010;34:757-761.

-3 Hurley TR, D’Angelo CM, Clasen RA, Wilkinson SB, Passavoy RD: Magnetic resonance imaging and pathological analysis of a pituicytoma: case report. Neurosurgery 1994;35:314-317.

-4 Brat DJ, Scheithauer BW, Staugaitis SM, Holtzman RN, Morgello S, Burger PC: Pituicytoma: a distinctive low-grade glioma of the neurohypophysis. Am J Surg Pathol 2000;24:362-368.

5 Suess V, Pliska V: Identification of the pituicytes as astroglial cells by indirect immunoflourescence staining for the glial fibrillary acid protein. Brain Res 1981;221:27-33.

-6 Figarella-Branger D, Dufour H, Fernandez C, Bouvier-Labit C, Grisoli F, Pellissier JF: Pituicytomas, a misdiagnosed benign tumor of the neurohypophysis; report of three cases. Acta Neuropathol 2002;104:313-319.

-7 Katsuta T, Inoue T, Nakagaki H, Takeshita M, Morimoto K, Iwaki T: Distinctions between pituicytoma and ordinary pilocytic astrocytoma. Case report. J Neurosurg 2003;98:404-406.

8 Kowalski RJ, Prayson RA, Mayberg MR: Pituicytoma. Ann Diagn Pathol 2004;8:290-294. 
-9 Ulm AJ, Yachins AT, Brat DJ, Rhoton AL Jr: Pituicytoma: report of two cases and clues regarding histogenesis. Neurosurgery 2004;54:753-757. 